\title{
ESOFAGOGASTRECTOMIA VÍDEO-ASSISTIDA PARA ADENOCARCINOMA DA TRANSIÇÃO ESOFAGOGÁSTRICA
}

\section{Video-assisted esophagogastrectomy in esophagastric transition adenocarcinoma}

\author{
André BRANDALISE, Nilton C. ARANHA, Nelson A. BRANDALISE, \\ Cláudia LORENZETTI, Conrado de Sousa PIRES
}

ABCDDV/547

Brandalise A, Aranha NC, Brandalise NA, Lorenzetti C, Pires CS. Esofagogastrectomia vídeo-assistida para adenocarcinoma da transição esofagogástrica. ABCD Arq Bras Cir Dig 2007;20(3):143-5.

RESUMO - Racional - Nas últimas décadas observou-se expressivo aumento na incidência de adenocarcinoma da transição esofagogástrica. Concomitante a este aumento, houve progresso na cirurgia minimamente invasiva. Objetivo - Relatar a experiência com a técnica de esofagectomia vídeo-assistida, transhiatal para o tratamento de adenocarcinoma da transição esofagogástrica. Métodos - Indicou-se a técnica em 30 pacientes, com predominância do sexo masculino (73\%) e idade média de 55 anos. Após avaliação laparoscópica, 19 pacientes foram operados por técnica vídeo-assistida. Resultados - O tempo cirúrgico variou de 4 a 5 horas. Houve necessidade de colocação de dreno torácico em 12 pacientes (63\%). Dois $(10,5 \%)$ apresentaram fístula na anastomose cervical. Houve um óbito devido à fistula intratorácica da linha de grampos. Excluindo este paciente, o estadio pós-operatóro mostrou três $(16,7 \%)$ pacientes no estadio I, dois (11\%) IIa, três $(16,7 \%)$ IIb, seis $(33,3 \%)$ III e quatro $(22,2 \%)$ no estadio IV. A sobrevida global média foi de 25,5 meses (3 a 105 meses), e quando separada em estádios, foi 53,3 meses até estádio IIa e 15,8 meses em estado IIb ou superior. Conclusão - A cirurgia minimamente invasiva pode ser alternativa no tratamento do adenocarcinoma da transição esofagogástrica.

DESCRITORES - Adenocarcinoma. Cirurgia vídeo-assistida. Esôfago.

\section{INTRODUÇÃO}

A incidência de adenocarcinoma do esôfago distal e cárdia vem crescendo muito nas últimas décadas nos países ocidentais. Em estudo publicado em 1991, analisando dados sobre a incidência de câncer em nove áreas dos Estados Unidos no período entre 1976 e 1987, observou-se incremento de $4 \%$ a $10 \%$ por ano - excedendo qualquer outro tipo de câncer ${ }^{1}$.

Em meados dos anos 80, metade das neoplasias gástricas eram adenocarcinomas de cárdia, e elas representavam um terço de todas as neoplasias de esôfago ${ }^{1}$. Em outro estudo, a incidência de adenocarcinoma da junção esofagogástrica subiu de 0,6 para 2,2 por 100.000 pessoas-ano comparando as décadas de 1971-1980 com 1991-2000². O aumento da obesidade nos paises ocidentais pode estar associado à essas observações ${ }^{3}$.

O presente estudo teve por objetivo relatar a experiência com a técnica de esofagectomia vídeo-assistida, transhiatal para o tratamento de adenocarcinoma da transição esofagogástrica.

Trabalho realizado no Gastrocentro, UNICAMP, Campinas, SP, Brasil.

Endereço para correspondência: André Brandalise, e-mail: andre@gastrosite.com.br

\section{MÉTODOS}

Foi feita análise retrospectiva dos casos de adenocarcinoma de cárdia operados pelos autores entre setembro de 1997 a maio de 2006. Nesse período, indicou-se ressecção por laparoscopia em 30 pacientes. Vinte e dois eram do sexo masculino (73\%) e a idade média era de 55 anos.

Segundo a classificação de Siewert, dividiram-se em oito casos do tipo I, 11 do tipo II e 11 do tipo III. Na abordagem inicial da cavidade, após analisarem-se fatores locais, decidiu-se mudar a via de acesso para laparotomia em 11 casos, para preservar a radicalidade oncológica. As neoplasias do tipo III foram as que mais fizeram mudar de tática operatória, sendo empregada a laparotomia em seis casos (54,5\%). Nos tipos I e II, o acesso laparoscópico foi mantido em $75 \%$ e $73 \%$ dos casos, respectivamente.

TABELA 1 - Tratamento aplicado frente à classificação de Siewert para o câncer de esôfago

\begin{tabular}{lccc}
\hline Classificação & Via de acesso $(\mathbf{n})$ & & Total \\
\hline \multirow{2}{*}{ Tipo I } & Vídeo-assistida & 6 & 8 casos \\
& Laparotomia & 2 & \\
\multirow{2}{*}{ Tipo II } & Vídeo-assistida & 8 & 11 casos \\
& Laparotomia & 3 & \\
\multirow{2}{*}{ Tipo III } & Vídeo-assistida & 5 & 11 casos \\
& Laparotomia & 6 & \\
\hline
\end{tabular}




\section{Técnica cirúrgica}

O paciente é posicionado em decúbito dorsal horizontal, com as pernas afastadas. O cirurgião fica entre elas, um auxiliar com a câmera à direita do paciente e outro à esquerda.

Inicia-se com o estadiamento da lesão e avaliação se o volume tumoral será empecilho para o procedimento laparoscópico. Tomada a decisão de continuar com a laparoscopia como via de acesso, aborda-se a grande curvatura com bisturi ultra-sônico, com cuidado para não perder a arcada gastroepiplóica de vista e acidentalmente ligá-la. Prossegue-se cranialmente com secção os vasos gástricos curtos, completando a liberação da grande curvatura.

Em seguida, os linfonodos hepáticos, tronco celíaco, e esplênicos são dissecados e retirados em separado. A artéria gástrica esquerda é ligada em sua origem.

Por último aborda-se a região da cárdia e o esôfago. Procura-se sempre remover todo o tecido adiposo/linfonodal regional em monobloco com a peça. Nesta fase, uma segunda equipe já está dissecando o esôfago cervical e seccionando-o, fixando a parte distal a uma sonda nasogástrica.

Através de mini-incisão, de tamanho suficiente para caber a mão do cirurgião para liberar aderências do esôfago médio, o esôfago e estômago são trazidos para fora da cavidade abdominal e um tubo gástrico estreito é confeccionado, com uso de grampeadores para operações laparotômicas e sepultamento da linha de grampos por sutura contínua com fio inabsorvível monofilamentar. A sonda é, então, fixada à ponta do tubo gástrico, que é tracionado para a região cervical.

Não é feita piloroplastia. A anastomose cervical é confeccionada com pontos separados e fio absorvível monofilamentar. Um dreno laminar fino é mantido na região cervical até a confirmação radiológica da ausência de fístula.

\section{RESULTADOS}

Analisando apenas os 19 pacientes submetidos à cirurgia vídeo-assistida, o tempo cirúrgico variou de 4 a 5 horas. Não houve sangramento que necessitasse transfusão em nenhum caso.

Os pacientes permaneceram apenas um dia na UTI nos pós-operatório e a permanência hospitalar média foi de 12 dias. Dois pacientes apresentaram fístula da anastomose cervical $(10,5 \%)$. Drenagem torácica foi realizada em 12 casos $(63 \%)$.

Houve um óbito intra-hospitalar, por fístula mediastinal da linha de grampeamento. Não houve mortalidade em 30 dias pós-operatório. No acompanhamento ambulatorial destes pacientes, $10(52,6 \%)$ queixaram-se de disfagia e foram submetidos à dilatação endoscópica da anastomose cervical.

O número médio de linfonodos ressecados foi 17 , variando de 6 a 28 .

De acordo com o estadio pós-operatório, os 18 pacientes sobreviventes, dividiram-se em: 3(16,7\%) em estadio I; 2(11\%) no IIa; $3(16,7 \%)$ no IIb; $6(33,3 \%)$ no III e $4(22,2 \%)$ no IV.
TABELA 2 - Estadiamente dos pacientes tratados

\begin{tabular}{lcc}
\hline TNM & Estadio & N (\%) \\
\hline Tis N0 M0 & I & $3(16,7 \%)$ \\
T1 N0 M0 & I & $2(11 \%)$ \\
T2 N0 M0 & IIa & \\
T1 N1 M0 & IIb & $3(16,7 \%)$ \\
T2 N1 M0 & IIb & \\
T3 N1 M0 & III & $6(33,3 \%)$ \\
T4 N1 M1 & III & \\
Tx Nx M1 & IV & $4(22,2 \%)$ \\
\hline
\end{tabular}

\section{DISCUSSÃO}

Desde $1993^{8}$, vários autores começaram a relatar sua experiência com a abordagem minimamente invasiva para esofagectomia.

DePaula et al. ${ }^{8}$ relataram, já em 1995, sua experiência com 48 esofagectomias, a maioria por megaesôfago grau IV.

Em seguida, passou-se a indicar a cirurgia minimamente invasiva também para neoplasias. Múltiplas técnicas foram propostas, entre elas a abordagem combinada por toracoscopia e laparoscopia, apenas por laparoscopia (transhiatal) e a acesso vídeo-assistido.

Os autores deste trabalho adotam, assim como outros ${ }^{10}$, o acesso vídeo-assistido por várias razões: a) visualização direta na dissecção das cadeias linfonodais do mediastino inferior que, por se tratarem geralmente da pacientes obesos, pode ser mais difícil por laparotomia; b) a mini-incisão permite inserir a mão no mediastino posterior para liberar aderências do terço médio do esôfago; c) é possível confeccionar um tubo gástrico mais estreito, diminuindo a estase alimentar; d) é possível fazer, facilmente, sutura de reforço sobre a linha de grampos; e) o custo dos grampeadores para laparotomia é bem menor que o de laparoscopia.

A via abdominal transhiatal é a preferida por apresentar baixa taxa de morbidade e mortalidade. Infelizmente, o único óbito desta série ocorreu em época em que não se fazia sutura de reforço sobre a linha de grampos da gastroplastia, mas desde então, não houve mais mortalidade ligada ao procedimento.

Em estudo recente, Morgan et al. ${ }^{4}$ compararam, prospectivamente, as taxas de sobrevida de esofagectomia transtorácia e transhiatal após terapia neoadjuvante para neoplasia de esôfago. Não houve diferença estatisticamente significante em relação à morbidade e mortalidade. Também a recorrência não foi mais freqüente em determinada técnica. A sobrevida em 5 anos foi $34 \%$ na transtorácica e $53 \%$ na transhiatal $(P=0,2298)$. Sobrevida média também foi similar nos pacientes com linfonodos acometidos (23 vs. 22 meses, transtorácia vs. transhiatal)

Fato interessante é que nesse estudo, a decisão em abordagem transtorácia ou transhiatal baseou-se no estágio da doença e no estado geral dos pacientes. Mesmo tendo os pacientes do grupo transhiatal performance mais baixa, morbidade, mortalidade, recorrência e sobrevida foram similares ao grupo transtorácico. 
Böttger et al. ${ }^{5}$, utilizando técnica combinada toracoscópica e transhiatal obteve, em média, 23 linfonodos (19-26) e 14 (12 a 17) quando usou exclusivamente a via transhiatal.

Sanders et al. ${ }^{6}$ publicaram os resultados de 18 pacientes tratados por cirurgia minimamente invasiva via transhiatal e o número de linfonodos ressecados variou de 2 a 26 (média de 10)

A média de linfonodos dissecados neste relato (17), está dentro do encontrado na literatura, mas acredita-se que este número pode ser maior, pois grande parte das peças foi dissecada apenas pelo patologista e não pelo próprio cirurgião, o que pode diminuir a busca por linfonodos.

Torres et al. ${ }^{7}$ demonstraram a importância da linfadenectomia na cirurgia para tratamento do esôfago, comparando grupos com linfadenectomia (feita por toracoscopia) e sem linfadenectomia (transhiatal). A sobrevida média em 5 anos nos pacientes sem envolvimento linfonodal foi $44 \%$ e $17 \%$ (com e sem linfadenectomia, respectivamente) e nos com envolvimento linfonodal, as taxas de sobrevida foram $21 \%$ e $6 \%$ (com e sem linfadenectomia, respectivamente).

No presente estudo, a sobrevida variou de 3 a 105 meses, com média de 25,5 meses, entretanto, quando se observa os pacientes com estadio até IIa, a sobrevida média foi de 53,3 meses, variando de 18 a 105 meses, com todos os pacientes vivos até o momento.
Já quando o estadio é IIb ou mais, a sobrevida média cai para 15,8 meses, variando de 3 a 47 meses, demonstrando que, além da penetração na parede, o envolvimento linfonodal está diretamente ligado ao prognóstico.

Nos pacientes com até três linfonodos acometidos, a sobrevida média foi de 73,6 meses, enquanto naqueles com metástase para mais de três, ela foi de 21,2 meses.

Geralmente, os casos selecionados para esofagectomia minimamente invasiva são os pequenos, e em estágios iniciais, mas mostra-se neste estudo que mesmo em neoplasias mais avançadas, a operaçao vídeo-assistida pode ser utilizada, sem prejuízo ao paciente, em comparação com a técnica aberta, desde que as condições locais assim o permitam. Estadiamento por laparoscopia, seguido por terapia neoadjuvante para "downstaging" pode permitir o acesso minimamente invasivo mesmo nestes casos, mas faltam estudos para avaliar a interferência nas taxas de sobrevida.

\section{CONCLUSÃO}

A cirurgia minimamente invasiva pode ser aplicada em neoplasias da transição esofagogástrica, sem prejudicar a radicalidade oncológica, em casos selecionados. A técnica vídeo-assistida não traz aumento nas complicações e pode diminuir os custos do procedimento.

Brandalise A, Aranha NC, Brandalise NA, Lorenzetti C, Pires CS. Video-assisted esophagogastrectomy in esophagogastric transition adenocarcinoma. ABCD Arq Bras Cir Dig 2007; 20(3):143-5.

ABSTRACT - Background - In the last decades an overwhelming increase in the incidence of esophagogastric transition adenocarcinomas has been observed. Jointly to this increase, there has also been progress in minimal invasive surgery. Aim - To relate the experience with video-assisted esophagectomy, transhiatal for the treatment of esophagogastric transition adenocarcinoma. Methods - The technique was indicated to 30 patients, predominantly male (73\%) with an average age of 55 years. After laparoscopic evaluation, 19 patients were operated on using the video-assisted technique. Results - Surgical time varied between 4 and 5 hours. Toracic drains had to be applied in 12 patients (63\%). Two (10,5\%) had cervical anastomosis fistulas. One death occurred due to intratoracic fistula in the clamp line. Excluding this patient, post-operative state demonstrated three $(16,7 \%)$ patients in stage I, two (11\%) in stage IIa, three $(16,7 \%)$ in IIb, six $(33,3 \%)$ in III and four $(22,2 \%)$ in stage IV. The overall life span was an average of 25,5 months (three to 105 months), and when categorized into stages, it reached 53,3 months up to stage IIa and 15,8 months in stage $\mathrm{IIb}$ or higher. Conclusion - Minimal invasive surgery can be an alternative in the treatment of esophagogastric transition adenocarcinoma.

HEADINGS - Adenocarcinoma. Video-assisted surgery. Esophagus.

\section{REFERÊNCIAS}

1. Blot WJ, Devesa SS, Kneller RW, Fraumeni JF Jr. Rising incidence of adenocarcinoma of the esophagus and gastric cardia. JAMA. 1991;265:1287-9.

2. Böttger T, Terzic A, Müller M, Rodehorst A. Minimally invasive transhiatal and transthoracic esophagectomy. Surg Endosc. 2007. (e-pub)

3. Braghetto IM, Burdiles PP, Korn OB. Esophagectomy and laparoscopic gastric mobilization with minilaparotomy for tubulization and esophageal replacement. Surg Eaparosc, Endosc Percutan Tech. 2001;11:119-25

4. Crane SJ, Richard Locke G, Harmsen WS, Diehl NN, Zinsmeister AR, Joseph Melton L, Romero Y, Talley NJ. The changing incidence of oesophageal and gastric adenocarcinoma by anatomic sub-site. Aliment Pharmacol Ther. 2007;25:447-53.

5. DePaula AL, Hashiba K, Ferreira EA, et al. Laparoscopic transhiatal esophagectomy with esophagogastroplasty. Surg Laparosc Endosc. 1995;5:1-5.
6. Merry AH, Schouten LJ, Goldbohm RA, van den Brandt PA. Merry, Schouten, Goldbohm, Van Den Brandt. Body mass index, height and risk of adenocarcinoma of the oesophagus and gastric cardia: a prospective cohort study. Gut. 2007. (e-pub ahead of print)

7. Morgan MA, Lewis WG, Hopper AN, Escofet X, Havard TJ, Brewster AE, Crosby TD, Roberts SA, Clark GW. Prospective comparison of transthoracic versus transhiatal esophagectomy following neoadjuvant therapy for esophageal cancer. Dis Esophagus. 2007;20:225-31.

8. Sanders G, Borie F, Husson E, Marie Blanc P, Gianluca Di Mauro1, Claus C, Millat B. Minimally invasive transhiatal esophagectomy: lessons learned. Surg Endosc. 2007. (e-pub)

9. Torres AJ, Sánchez-Pernaute A, Hernando F, Díez Valladares L, González López O, Pérez Aguirre E, Suárez A, Balibrea JL. Two-field radical lymphadenectomy in the treatment of esophageal carcinoma. Dis Esophagus. 1999;12:137-43. 